\title{
Exchange-spring behavior below the exchange length in hard-soft bilayers in multidomain configurations
}

\author{
A. Quesada, ${ }^{1, *}$ G. Delgado, ${ }^{2}$ L. Pascual,${ }^{3}$ A. M. Aragón, ${ }^{4}$ P. Marín,,${ }^{4}$ C. Granados-Miralles,${ }^{1}$ M. Foerster, ${ }^{5}$ L. Aballe ${ }^{5}$ \\ J. E. Prieto, ${ }^{6}$ J. de la Figuera, ${ }^{2}$ J. F. Fernández, ${ }^{1}$ and P. Prieto ${ }^{7}$ \\ ${ }^{1}$ Instituto de Cerámica y Vidrio (CSIC), Madrid 28049, Spain \\ ${ }^{2}$ Instituto de Química Física “Rocasolano” (CSIC), Madrid 28006, Spain \\ ${ }^{3}$ Instituto de Catálisis y Petroleoquímica (CSIC), Madrid 28049, Spain \\ ${ }^{4}$ Instituto de Magnetismo Aplicado (UCM-ADIF), Las Rozas, Madrid 28230, Spain \\ ${ }^{5}$ ALBA Synchrotron Light Facility, CELLS, Barcelona 08290, Spain \\ ${ }^{6}$ Centro de Microanálisis de Materiales (CMAM) and Departamento Física de la Materia Condensada, \\ Universidad Autónoma de Madrid, 28049 Madrid, Spain \\ ${ }^{7}$ Departamento Física Aplicada, Universidad Autónoma de Madrid, 28049 Madrid, Spain
}

(Received 17 May 2018; revised manuscript received 15 November 2018; published 21 December 2018)

\begin{abstract}
Exchange-coupled hard-soft biphase magnets are technologically relevant systems in that they enable tailoring the magnetization reversal process. Here, exchange-spring behavior is observed in $\mathrm{CoFe}_{2} \mathrm{O}_{4} / \mathrm{FeCo}$ bilayers for soft thicknesses as thin as $2 \mathrm{~nm}$, at least four times below the exchange length of the system. This result is in contrast with the accepted theory for spring magnets that states that the exchange length defines the critical thickness below which both magnetic phases should be rigidly coupled. In combination with micromagnetic calculations, this surprising observation is understood as a consequence of the dominance of domain-wall propagation in the soft phase during the reversal process, so far unaccounted for in theoretical descriptions. Our results emphasize the need to expand the existing spring theory from coherent rotation to domain-wall related processes in multidomain configurations in order to accurately design magnetic heterostructures with controllable reversal.
\end{abstract}

DOI: 10.1103/PhysRevB.98.214435

\section{INTRODUCTION}

In the quest for novel and improved materials, multiphase nanostructures bring forward the opportunity to combine in a single material different properties and functionalities. Notably, bimagnetic nanostructures consisting of a hard (high anisotropy) and a soft (high magnetization) phase provide an additional degree of freedom-the reversal behaviorwhich offers a broader range of possibilities for tailoring the magnetic response of the system. Examples include coreshell nanoparticles [1], thin bilayer films [2], and nanostructured ceramic materials [3] with applications in a variety of fields such as catalysis, permanent magnets [4,5], information storage [6,7], and biomedicine [8].

The particular case of exchange-coupled bilayer thin films can be exploited for improving the writability in magnetic recording media without compromising thermal stability, and constitutes an extremely interesting model system for understanding magnetization reversal processes in exchangecoupled magnets $[9,10]$. The size of the soft phase dictates the magnetization switching behavior, with the existence of a critical size below which the soft phase is rigidly exchange coupled to the hard phase and both reverse simultaneously $[2,11,12]$. Above the critical size, but for moderate thicknesses, the soft phase switches reversibly at lower fields than the hard one through an exchange-spring process. For large

\footnotetext{
*Corresponding author: a.quesada@icv.csic.es
}

soft thickness, the system is considered decoupled and the two phases reverse independently. Theory predicts that the critical thickness separating the rigid and spring regimes does not deviate significantly from the exchange length of the hard phase, i.e., the Bloch wall width, which is usually of the order of a few nanometers [2,13-15].

The critical thicknesses observed in experimental systems based on uniaxially anisotropic hard phases such as FePt, $\mathrm{SmCo}_{5}$, and $\mathrm{Nd}_{2} \mathrm{Fe}_{14} \mathrm{~B}$ are in reasonably good agreement with theoretical models $[2,15,16]$. Even systems based in biaxial $\mathrm{CoFe}_{2} \mathrm{O}_{4}$ (CFO) with $\mathrm{Fe}_{3} \mathrm{O}_{4}$ as soft layer accurately follow the predictions [17]. Nonetheless, the complexity of real systems is extremely hard to fully model and experimental critical thicknesses are often slightly overestimated by the models $[16,18]$. So far, these deviations have been moderate at most and thus the models have predicted reversal behaviors with reasonable accuracy.

Previous works on $\mathrm{CoFe}_{2} \mathrm{O}_{4} / \mathrm{FeCo}$ bilayers have not focused on studying the critical thickness of the system. In Sayed Hassan et al. [19] and Viart et al. [20], spring behavior is observed for soft layers as thin as $5 \mathrm{~nm}$ in polycrystalline films. A diversity of magnetic coupling behaviors is reported as a function of the preparation conditions, including decoupling, exchange coupling, and antiferromagnetic coupling. No rigid coupling is detected; in all cases spring-type or independent magnetization reversal occurs [19-22].

Here we investigate magnetization reversal in $\mathrm{CoFe}_{2} \mathrm{O}_{4} / \mathrm{FeCo}$ exchange-coupled films as a function of the soft layer thickness, in an attempt to determine the 
critical thickness separating rigid and spring behavior. The bilayers are observed in transmission electron microscopy (TEM), while Rutherford backscattering spectrometry (RBS) is employed to confirm the composition and hard/soft thicknesses. The magnetic characterization reveals spring behavior for all soft thicknesses studied, down to $2 \mathrm{~nm}$, well below the exchange length of the system (approximately $8 \mathrm{~nm}$ ). The physical mechanisms behind this surprising behavior are discussed based on the experimental data and supported by micromagnetic calculations.

\section{EXPERIMENTAL}

The $\mathrm{CFO} / \mathrm{FeCo}$ bilayers were deposited on TiN buffered $\mathrm{Si}$ (001) substrates by $\mathrm{Ar}^{+}$ion-beam sputtering. Prior to deposition, the native $\mathrm{SiO}_{2}$ was removed by dipping the substrate into a $5 \% \mathrm{HF}$ solution for $2 \mathrm{~min}$. A $99.9 \%$ pure TiN target was used to deposit the TiN buffer while $99.9 \%$ pure ceramic $\mathrm{CoFe}_{2} \mathrm{O}_{4}$ and metallic $\mathrm{Fe}_{1.3}$ Co commercial targets were used to deposit the hard CFO films and the soft $\mathrm{FeCo}$ metallic layer, respectively. The samples were capped with 2-nm aluminum. The TiN and $\mathrm{CoFe}_{2} \mathrm{O}_{4}$ films were deposited at a constant substrate temperature of $400{ }^{\circ} \mathrm{C}$ while the soft metallic layer was deposited at $200^{\circ} \mathrm{C}$ to prevent oxygen diffusion. A vacuum chamber with a base pressure of $2 \times 10^{-5} \mathrm{~Pa}$ was used. During deposition the pressure was maintained at $3.3 \times 10^{-2} \mathrm{~Pa}$ and the substrates were rotated at $2 \mathrm{rpm}$ to increase the homogeneity of the deposit. An oxygen partial pressure, $P_{\mathrm{O} 2}=3 \times 10^{-3} \mathrm{~Pa}$, was kept to deposit the $\mathrm{CoFe}_{2} \mathrm{O}_{4}$ layer. All CFO layers were grown up to the same nominal thickness as indicated by a quartz balance. TEM experiments were conducted on a field emission gun JEOL $2100 \mathrm{~F}$ operating at $200 \mathrm{kV}$ and equipped with a scanning transmission electron microscopy (STEM) unit with both bright and dark field detectors. Energy dispersive $\mathrm{x}$-ray spectroscopy (EDXS) maps were acquired in high angle annular dark field (HAADF)-STEM mode with an Oxford INCA X-sight system. In-depth composition and thickness of the different layers were determined by RBS at the Centro de Microanálisis de Materiales 5-MV tandem accelerator using ${ }^{4} \mathrm{He}^{+}$at $1.8 \mathrm{MeV}$. The elemental distribution and thickness quantification of the different layers were determined with the SIMNRA simulation software package. The magnetic properties were measured using a vibrating sample magnetometer (VSM, Quantum Design PPMS) with the field applied along an easy CFO inplane axis. The diamagnetic signal from the $\mathrm{Si}(001)$ substrate was subtracted. The angular dependence of magnetization
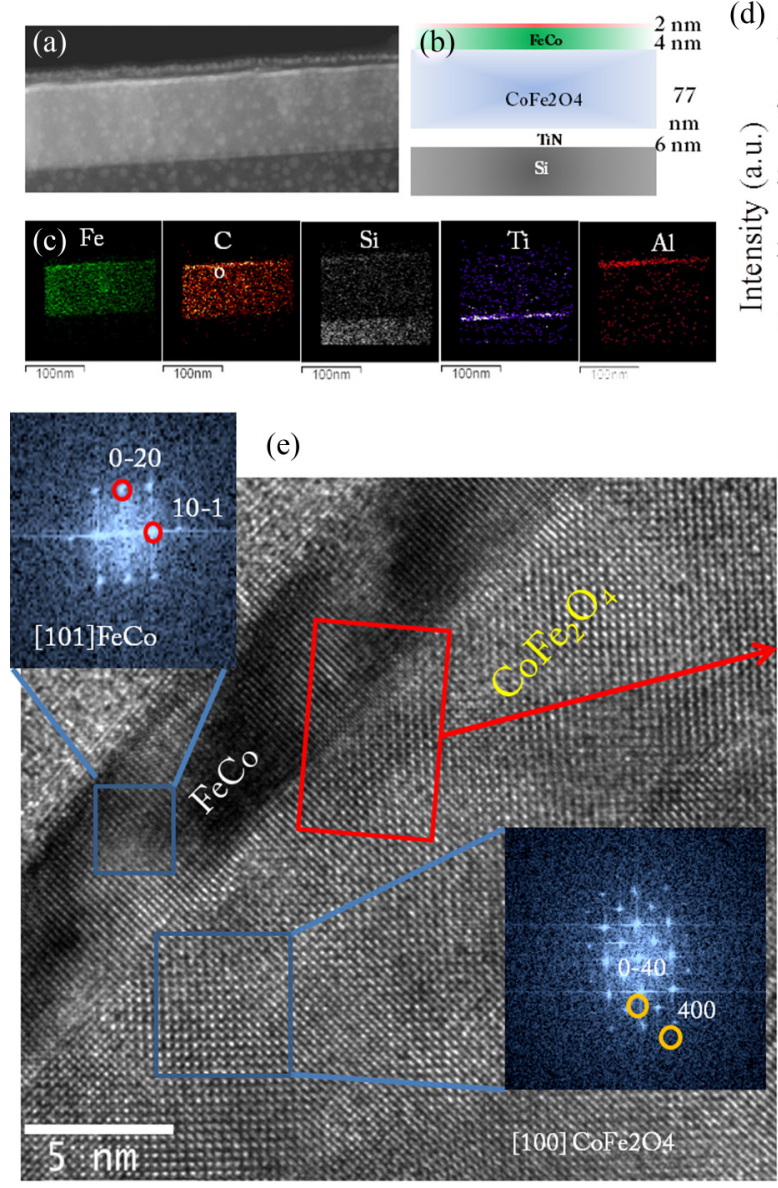

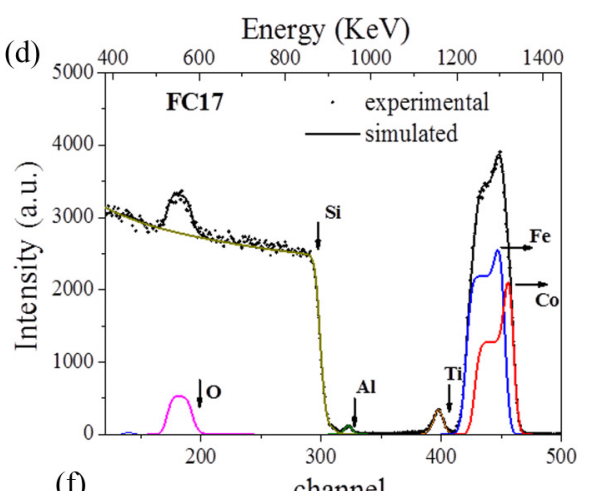

(f)

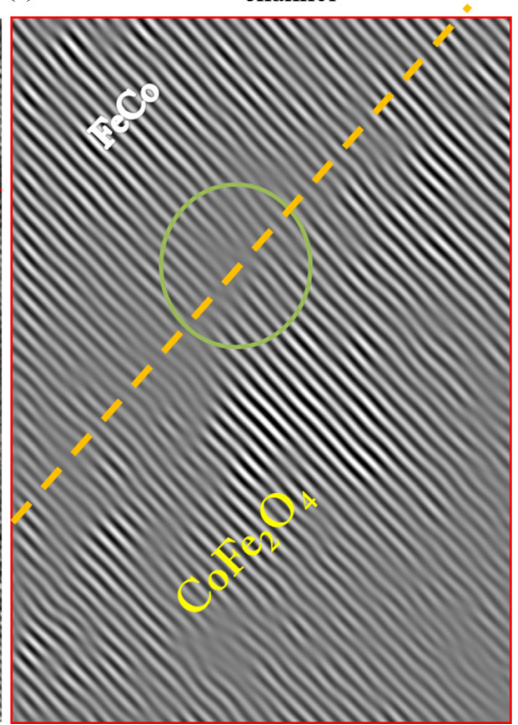

FIG. 1. TEM characterization. (a) Low resolution STEM-HAADF image showing the multilayer arrangement sketched in (b). (c) EDXS maps for different elements present in the stack. (d) RBS data corresponding to a bilayer with soft FeCo thickness of $17 \mathrm{~nm}$. A HRTEM image of the $\mathrm{CFO} / \mathrm{FeCo}$ interface is shown in (e), with insets containing fast Fourier-transform patterns for each film. In (f), a filtered image using together the (10-1) in FeCo and (400) in CFO of the interface region delimited in red is presented. 
minor loops was investigated at room temperature by high resolution vectorial-Kerr magneto-optical measurement (MOKE) in a longitudinal configuration. MOKE hysteresis loops were recorded by changing the in-plane angular orientation of the sample, keeping fixed the external magnetic field direction. The angular orientation, ranging from $0^{\circ}$ to $360^{\circ}$, was probed at intervals of $9^{\circ}$ with a maximum applied magnetic field of $0.1 \mathrm{~T}$. The in-plane size of the simulation cell is $4 \times 4 \times 2 \mathrm{~nm}^{3}$ for both layers and several replicas were made to avoid isolated system behavior. The thicknesses of $\mathrm{CFO}$ and $\mathrm{FeCo}$ were 77 and $4 \mathrm{~nm}$, respectively. The exchange stiffness and anisotropy values were extracted from the literature $[18,23,24]$, while the saturation magnetization was taken from the experimental measurements. The following set of magnetic parameters was thus used as input in the simulations: exchange stiffness of hard $(H)$ and soft $(S)$ phases $A_{s}(\mathrm{CFO})=1.10 \times 10^{-11} \mathrm{~J} \mathrm{~m}^{-1}$ and $A_{s}(\mathrm{FeCo})=$ $1.7 \times 10^{-11} \mathrm{~J} \mathrm{~m}^{-1}$, respectively; saturation magnetization $M_{s}(\mathrm{CFO})=3.41 \times 10^{5} \mathrm{~A} \mathrm{~m}^{-1}$ and $M_{s}(\mathrm{FeCo})=1.85 \times$ $10^{6} \mathrm{~A} \mathrm{~m}^{-1}$, respectively; and magnetocrystalline constant $K_{H}=5.10 \times 10^{5} \mathrm{~J} \mathrm{~m}^{-3}$ and $K_{S}=-0.472 \times 10^{3} \mathrm{~J} \mathrm{~m}^{-3}$, respectively). Magnetization curves were calculated and plotted for different initial domain configurations (single and multidomain states). The multidomain state was achieved by defining $100 \times 100$-nm squares in the CFO layer that are exchange decoupled from one another. Different degrees of exchange coupling at the interface were investigated, ranging from one (perfect coupling) to zero (complete decoupling) [3]. $\mathrm{X}$-ray magnetic circular dichroism-photoemission electron microscopy (XMCD-PEEM) experiments were performed at the CIRCE beamline of the ALBA Synchrotron [25]. The instrument allows acquiring XMCD images to space resolve in remanence the in-plane magnetization component for a given atomic element, $\mathrm{Fe}$ in this paper, along the $\mathrm{x}$-ray direction. To determine the domain-wall width from the images, the usual criterion of measuring the distance between the $85-15 \%$ intensity change in a line profile was employed [25]. Micromagnetic simulations were performed using the Mumax III [26] on a system composed of two layers: CFO (hard material) and FeCo alloy (soft material) on top.

\section{RESULTS}

Figure 1(a) shows a STEM-HAADF image of the complete layer stack-sketched in Fig. 1(b) - from which the different thicknesses can be calculated. The multilayer includes, sequentially, a 2-nm Al capping layer, $4.1 \mathrm{~nm}$ FeCo, $77 \mathrm{~nm}$ CFO, a 6-nm TiN buffer layer, and $\mathrm{Si}$ (001). This sample will be later referred to as FC4, responding to the thickness of the soft phase layer. In Fig. 1(c), EDXS maps confirm the composition of each layer. As expected, a higher $\mathrm{Fe}$ and $\mathrm{Co}$ intensity is observed in the metallic FeCo layer with respect to CFO. Figure 1(e) shows a HRTEM image of the $\mathrm{CFO} / \mathrm{FeCo}$ interface. The fast Fourier transform for each film confirms the cubic spinel and bcc structure of the $\mathrm{CFO}$ and $\mathrm{FeCo}$ films, respectively, allowing us to infer an epitaxial relationship [101] FeCo // [100] CFO between the layers, in agreement with previous reports [22].

The image filtered by the (10-1) FeCo and (400) CFO diffraction spots, shown in Fig. 1(f), demonstrates a robust
TABLE I. Hard and soft layer thicknesses of all samples extracted from RBS.

\begin{tabular}{lcc}
\hline \hline Sample & FeCo thickness $(\mathrm{nm})$ & CFO thickness $(\mathrm{nm})$ \\
\hline CFO & & 77.2 \\
FC2 & 2.0 & 81.1 \\
FC4 & 4.0 & 77.2 \\
FC7 & 6.8 & 77.8 \\
FC13 & 13.1 & 81.4 \\
FC17 & 17.1 & 77.2 \\
\hline \hline
\end{tabular}

degree of structural coherency at the interface between both phases, with the exception of occasional dislocations as the one indicated with the green circle.

Figure 1(d) presents the RBS spectrum corresponding to the FC17 sample. Using the data obtained for all samples, the hard and soft thicknesses are calculated and summarized in Table I.

Figure 2(a) shows the magnetization curves of FC2, FC4, and the reference $\mathrm{CFO}$ sample. $\mathrm{CFO}$ presents a saturation magnetization of $M_{s}(\mathrm{CFO})=3.41 \times 10^{5} \mathrm{~A} \mathrm{~m}^{-1}$, slightly smaller than the usual bulk value, presumably due to the strain from the substrate [17]. The squareness ratio is $M_{r} / M_{s}=$ 0.46 , indicating that the hard phase breaks into a multidomain pattern. For samples FC2 and FC4, the squareness ratios are 0.43 and 0.64 , indicative as well of a multidomain configuration at remanence. However, they portray a very different magnetization curve. At low negative fields, a steep decrease in magnetization is observed, associated with the reversal of the soft phase. At higher fields the gradual irreversible rotation in CFO occurs. The onset of soft layer reversal at low negative fields is defined as the nucleation field $H_{n}$, while the switching field $H_{\mathrm{sw}}$ corresponds to the irreversible magnetization reversal of the hard layer [17,27]. $H_{n}$ and $H_{\text {sw }}$ are extracted from the magnetization curves as the inflection points in the soft and hard reversal parts of the demagnetization curve, respectively [17], as indicated with arrows in Fig. 2(a). This reversal behavior demonstrates nonrigid coupling as hard and soft layers do not reverse simultaneously. Nonrigidity is observed when $H_{n}<H_{\mathrm{sw}}$ [17], a condition that is fulfilled for all soft thicknesses as shown in Fig. 2(c).

Even if $H_{n}$ depends on all relevant magnetic parameters of both hard and soft phases [27], it is often ultimately determined by the exchange stiffness $A_{s}$, saturation magnetization $M_{s}$, and thickness $t_{s}$ of the soft phase, according to Eq. (1) $[2,12,16]$ :

$$
H_{n}=\frac{\pi^{2} A s}{2 M s t s^{2}} .
$$

Using the experimentally determined value of $M_{s}(\mathrm{FeCo})=1.85 \times 10^{6} \mathrm{~A} \mathrm{~m}^{-1}$ and the accepted stiffness value of $A_{s}=1.7 \times 10^{-11} \mathrm{~J} \mathrm{~m}^{-1}[18,24]$, the theoretical evolution of $H_{n}$ as a function of soft thickness has been calculated using Eq. (1) and is plotted in Fig. 2(c). A deviation between theoretical and experimental values of $H_{n}$ is clearly observed. The critical point at which $H_{n}$ (theoretical $)=H_{\text {sw }}$ corresponds to a 8-nm soft layer, which is in agreement with the accepted theory on spring magnets $[2,28]$ : the critical 

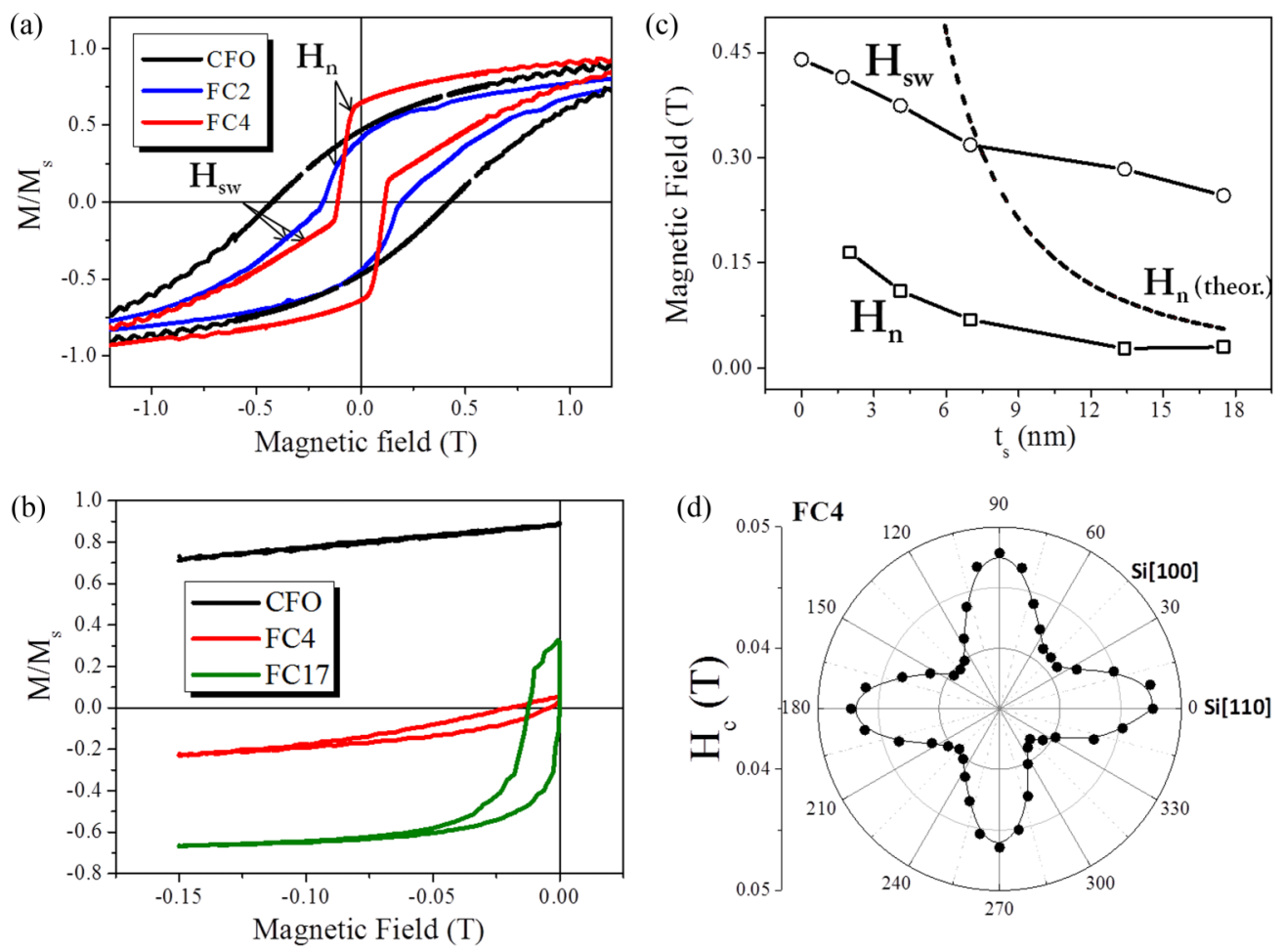

FIG. 2. (a) VSM magnetization curves measured at room temperature of a reference CFO film for a 2-nm (FC2) and a 4-nm soft bilayer (FC4). (b) Recoil loops of FC4, FC17, and CFO measured after saturating at $+5 \mathrm{~T}$. (c) $H_{n}$ and $H_{\mathrm{sw}}$ extracted from the magnetization curves as a function of soft thickness. (d) Polar plot of coercivity $\left(H_{c}\right)$ determined by MOKE as a function of the in-plane angle between the applied field and the (110) direction of the Si substrate for the FC4 sample.

soft thickness below which rigid reversal should occur is predicted to be close to the Bloch wall width $\delta_{w}$ of the hard phase, which for the case of CFO is of the order of $8-10 \mathrm{~nm}$ [17].

It is important to emphasize that even if both layers are not reversing simultaneously as in a rigidly coupled system they are not decoupled, i.e., they behave as a spring magnet. The decrease of $H_{n}$ with soft thickness is a strong indication of exchange coupling; in a fully decoupled situation the soft layer would always reverse at the same field. In order to corroborate the spring behavior, recoil loops were measured using a recoil field, $H_{R}$, which fulfills the condition $H_{\text {sw }}>H_{R}>H_{n}$. Figure 2(b) shows normalized recoil loops of CFO, FC4, and FC17 samples, obtained after saturating at $+5 \mathrm{~T}$ and measuring a loop between $H_{R}=-0.15$ and 0 T. For CFO, a closed loop with rather small magnetization recovery is observed [29]. From the FC4 and FC17 loops, we infer that both the openness and the recovered magnetization increase with the soft thickness, in agreement with previous observations [30-33]. FC17 in particular shows a remarkable recovery of magnetization from -0.66 to 0.31 and a change in slope for fields smaller than $0.03 \mathrm{~T}$, i.e., below the $H_{n}$ of FC17. This behavior clearly reveals the spring mechanism, with the unreversed hard layer rotating a large fraction of the soft spins back to their original direction at low fields, and allows us to discard decoupling for soft thicknesses up to $17 \mathrm{~nm}[29,30,33-35]$.

In addition, a robust degree of coupling is strongly supported by the angular dependence of $H_{c}$ evidenced by MOKE experiments presented in Fig. 2(d). MOKE is more sensitive to the surface and the applied magnetic field is $0.1 \mathrm{~T}$, which is below $H_{\mathrm{sw}}$, thus the main contribution to the maxima observed comes from the FeCo layer. From the angular dependence, it can be inferred that the FeCo easy axis, like CFO, is along Si[110] [34], when it should be along Si[100] based on the epitaxial relationship. This evidences the alignment of the hard and soft easy axes and proves significant exchange coupling at the interface.

XMCD-PEEM images were obtained in order to reveal the magnetic domain structure of the pure CFO film. The image, shown in the inset of Fig. 3(a), shows a multidomain configuration in the as-grown remanent state, composed of relatively small domains with sizes in the 100-500-nm range. In the profile plotted in Fig. 3(a), the domain-wall width is measured at $\delta_{w}=17.4 \mathrm{~nm}$. However, the error in the determination of $\delta_{w}$ is large, as the lateral resolution of the instrument is $15-20 \mathrm{~nm}$ [25], and thus a smaller $\delta_{w}$ cannot be discarded. Nonetheless, the standard structural and magnetic properties of the CFO films do not suggest abnormally small $\delta_{w}$ values and seem to indicate that we are observing spring reversal at thicknesses at least four times lower than predicted by the theory.

In the following, we will discuss the reasons for this surprising spring behavior. It is important to stress that spring magnet models have been almost exclusively developed based on uniaxial single-domain hard phases and considering coherent rotation as the demagnetization mechanism $[2,11,13,16]$. More sophisticated models that account for domain-wall nucleation in the soft layer, propagation towards the interface, and depinning onto the hard layer 

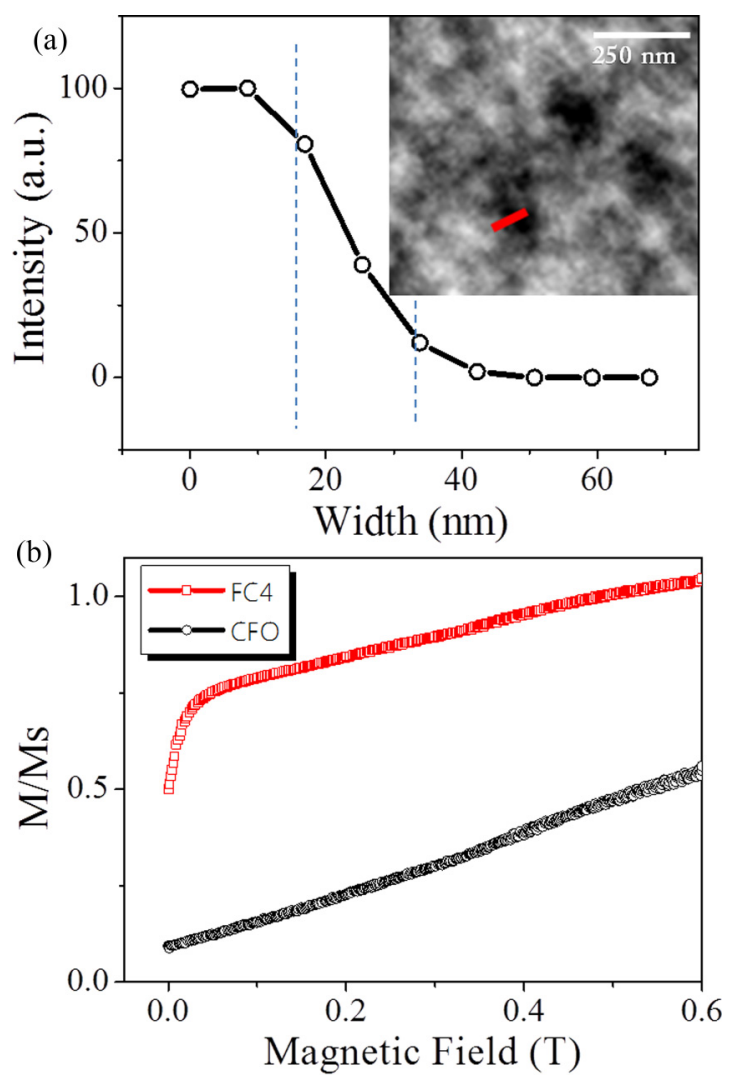

FIG. 3. (a) Profile corresponding to the magnetic domain wall indicated by the red line. Inset: XMCD-PEEM image obtained at the Fe L edge at remanence and RT showing magnetic domains in the pure CFO film. (b) First magnetization (virgin) curves for CFO and FC4.

have found that the critical thickness may be slightly lower than $\delta_{w}[4,26]$. However, to our knowledge, no theoretical description includes domain-wall propagation (DWP) in a multidomain configuration. DWP is activated at significantly lower fields than nucleation and rotation processes. Asti et al. [16] acknowledged that the slightly lower experimental $H_{n}$ values observed in some cases may be due to neglecting domain-wall propagation in the models, which Goto et al. already considered "an oversimplification" more than 50 years ago [12].

The shape of the first magnetization (or virgin) curve gives information about the dominant magnetization mechanism: propagation or nucleation [36]. The virgin curves of FC4 and CFO are shown in Fig. 3(b). For the single CFO layer, a nucleation/pinning dominated process is observed to gradually magnetize the film, that can be associated to domain-wall pinning at the antiphase boundaries of the CFO layer [17]. In comparison, the sharp increase in magnetization taking place at fields below $H_{n}$ in FC4 allows us to infer that a DWP process is quickly magnetizing the soft layer, strongly suggesting that DWP within the soft layer is the key factor contributing to the decrease of $H_{n}$.

Within the accepted spring theory based on domain nucleation, there are other parameters that could lead to a reduced critical thickness: reduced exchange stiffness, imperfect exchange coupling at the interface, and magnetic surface/interface anisotropies. However, in order to explain our observations, highly unrealistic values have to be considered, as addressed below for each case.

Figure 4 shows the simulated magnetization curves and their derivative for a bilayer analogous to FC4 in thicknesses and composition for four different exchange stiffness values. A single-domain initial configuration is defined in these simulations. At low negative fields, a maximum is clearly observed in Fig. 4(a) for $A_{s}=3 \cdot 1 \cdot 10^{-12} \mathrm{~J} / \mathrm{m}$, that corresponds to the nucleation field $H_{n}$ of the soft phase, as can be confirmed in Fig. 4(b). For $A_{s}=7 \cdot 10^{-12} \mathrm{~J} / \mathrm{m}$ the maximum has almost disappeared, hinting at a residual spring reversal of the soft layer. For larger $A_{s}$ values, the bilayer is seen to reverse as a rigid magnet, as the red curve in Fig. 4(b) demonstrates. Thus, micromagnetic calculations shown in Fig. 4 confirm that the system behaves as a $\mathrm{rigid} /$ spring magnet above/below $A_{s}=8 \times 10^{-12} \mathrm{~J} \mathrm{~m}^{-1}$. It is, however, extremely unrealistic to expect such low stiffness. Substituting in Eq. (1) the experimental values extracted from the magnetization curves for $H_{n}$ and $M_{s}\left[M_{s}(\mathrm{FeCo})=\right.$ $1.85 \times 10^{6} \mathrm{~A} \mathrm{~m}^{-1}$ ], we obtain $A_{s}=1.1 \times 10^{-12} \mathrm{~J} \mathrm{~m}^{-1}$ for $\mathrm{FC} 1$ and $A_{s}=3.1 \times 10^{-12} \mathrm{~J} \mathrm{~m}^{-1}$ for FC4. These values are an order of magnitude smaller than the theoretical accepted value for $\mathrm{Fe}_{65} \mathrm{Co}_{35}$ of $A_{s}=1.7 \times 10^{-11} \mathrm{~J} \mathrm{~m}^{-1}$. Further details on the unphysical $A_{s}$ needed to explain the behavior are given in the Supplemental Material [37].

Imperfect exchange coupling is known to lead to a decrease in critical thickness as well [11], and it is important to keep in mind that we are dealing with a metal/oxide interface, with potential roughness, mismatch, and/or interface oxidation issues. The occasional dislocations observed in Fig. 1(f) lead to an imperfect exchange coupling as structural coherency is disrupted. However, a relatively well-defined interface and structural coherency are observed in HRTEM and a robust degree of coupling has been experimentally demonstrated, indicating a limited dislocation-induced hindering of interfacial exchange. The simulated magnetization curves presented in the Supplemental Material [37] demonstrate that, for the accepted $A_{s}=1.7 \times 10^{-12} \mathrm{~J} \mathrm{~m}^{-1}$, the exchange-coupling parameter $\kappa$ would have to be as low as $\kappa=0.05$ in order to observe spring behavior $(\kappa=1 / \kappa=0$ for perfect coupling/decoupling), while we can safely assume $\kappa>0.5$ in our bilayers.

Although surface and interface anisotropies may be of relevance as well [38], their influence on nucleation fields is restricted to perpendicularly magnetized bilayers $[24,38]$ and are thus not relevant here.

Finally, the important differences between our results and the recent work by Lavorato et al. [17]—where $\mathrm{CFO} / \mathrm{Fe}_{3} \mathrm{O}_{4}$ exchange-coupled bilayers were studied-are addressed. An excellent agreement between the critical thickness derived from theoretical models - that do not account for DWPand their experimental value is observed in Ref. [17]. An important difference is that $M s$ is 2.5 times larger in $\mathrm{FeCo}$ and simulated magnetization curves shown in Fig. 4 prove that the reversal changes from rigid to spring mode as $M s$ increases. In addition, $\mathrm{Fe}_{3} \mathrm{O}_{4}$ thin films usually present a large density of antiphase boundaries, a structural defect that prevents DWP $[39,40]$. 

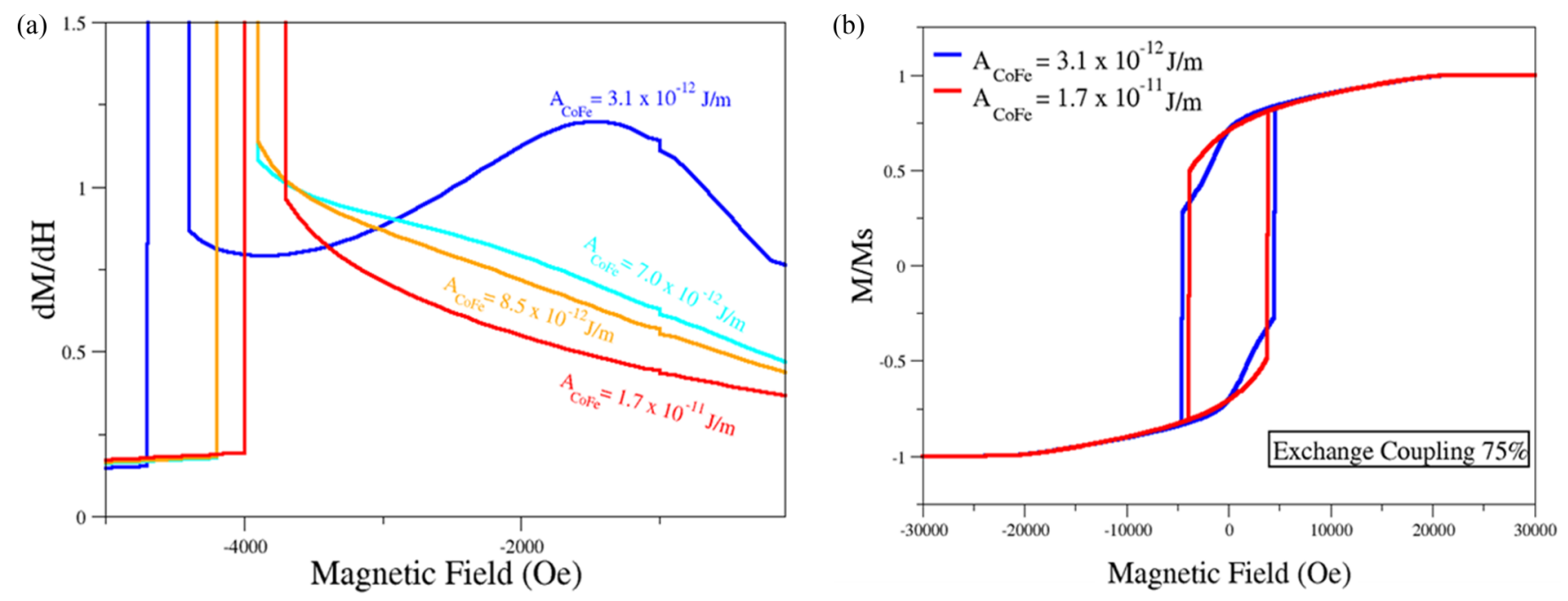

FIG. 4. (a) Simulated $d M / d H$ curve for four different exchange stiffness values for a 4-nm $\mathrm{FeCo} / 77-\mathrm{nm} \mathrm{CoFe}_{2} \mathrm{O}_{4}(\mathrm{CFO})$ bilayer. (b) Simulated magnetization curves corresponding to the highest and lowest stiffness values.

\section{CONCLUSIONS}

In summary, spring-type magnetization reversal is observed in hard-soft $\mathrm{CoFe}_{2} \mathrm{O}_{4} / \mathrm{FeCo}$ exchange-coupled bilayers for soft thicknesses as low as $2 \mathrm{~nm}$. This size is four times smaller than the critical thickness predicted by theoretical models, determined by the exchange length of the hard phase. This strong deviation is understood as a consequence of domain-wall propagation processes in the soft layer that govern its spring-type reversal, and are unaccounted for in the accepted theory for spring magnets. This observation could be relevant for understanding the reasons for the disappointing energy product values reached so far in advanced permanent magnets based on exchange-coupled composites. More importantly, our results bring forward the need to account for multidomain configurations and domain-wall propagation in the models for exchange-coupled multilayers in order to engineer their demagnetization curve, with important implications in the development of magnetic recording media and magnetic heterostructures in general.

\section{ACKNOWLEDGMENTS}

This work is supported by the Spanish Ministerio de Ciencia, Innovación y Universidades through Projects No. MAT2017-86450-C4-1-R, No. MAT2015-64110-C2-1-P, No. MAT2015-64110-C2-2-P, and No. FIS2017-82415-R and by the European Commission through H2020 Project No. 720853 (AMPHIBIAN) and FP7 Project No. 310516 (NANOPYME). Some of the experiments were performed at the CIRCE beamline of the ALBA Synchrotron Light Facility.
[1] A. López-Ortega, M. Estrader, G. Salazar-Alvarez, A. G. Roca, and J. Nogués, Phys. Rep. 553, 1 (2015).

[2] E. E. Fullerton, J. S. Jiang, and S. D. Bader, J. Magn. Magn. Mater. 200, 392 (1999).

[3] A. Quesada, C. Granados-Miralles, A. López-Ortega, S. Erokhin, E. Lottini, J. Pedrosa, A. Bollero, A. M. Aragón, F. Rubio-Marcos, M. Stingaciu, G. Bertoni, C. de Julián Fernández, C. Sangregorio, J. F. Fernández, D. Berkov, and M. Christensen, Adv. Electron. Mater. 2, 1500365 (2016).

[4] O. Gutfleisch, M. A. Willard, E. Brück, C. H. Chen, S. G. Sankar, and J. P. Liu, Adv. Mater. 23, 821 (2011).

[5] D. Sander, S. O. Valenzuela, D. Makarov, C. H. Marrows, E. E. Fullerton, P. Fischer, J. McCord, P. Vavassori, S. Mangin, P. Pirro, B. Hillebrands, A. D. Kent, T. Jungwirth, O. Gutfleisch, C. G. Kim, and A. Berger, J. Phys. D. 50, 363001 (2017).

[6] A. Hirohata, H. Sukegawa, H. Yanagihara, I. Zutic, T. Seki, S. Mizukami, and R. Swaminathan, IEEE Trans. Magn. 51, 1 (2015).
[7] R. L. Stamps, S. Breitkreutz, J. Åkerman, A. V Chumak, Y. Otani, G. E. W. Bauer, J.-U. Thiele, M. Bowen, S. A. Majetich, M. Kläui, I. L. Prejbeanu, B. Dieny, N. M. Dempsey, and B. Hillebrands, J. Phys. D 47, 333001 (2014).

[8] E. Fantechi, C. Innocenti, M. Zanardelli, M. Fittipaldi, E. Falvo, M. Carbo, V. Shullani, L. Di Cesare Mannelli, C. Ghelardini, A. M. Ferretti, A. Ponti, C. Sangregorio, and P. Ceci, ACS Nano 8, 4705 (2014).

[9] P. L. Francesca Casoli, Lucia Nasi, and Franca Albertini, in Ultra-High-Density Magnetic Recording: Storage Materials and Media Designs, edited by F. C. Gaspare Varvaro (Pan Standford, Singapore, 2016), p. 48.

[10] D. Makarov, J. Lee, C. Brombacher, C. Schubert, M. Fuger, D. Suess, J. Fidler, and M. Albrecht, Appl. Phys. Lett. 96, 062501 (2010).

[11] G. Asti, M. Solzi, and M. Ghidini, J. Magn. Magn. Mater. 226230, 1464 (2001).

[12] E. Goto, N. Hayashi, T. Miyashita, and K. Nakagawa, J. Appl. Phys. 36, 2951 (1965). 
[13] M. Ghidini, G. Asti, R. Pellicelli, C. Pernechele, and M. Solzi, J. Magn. Magn. Mater. 316, 159 (2007).

[14] G. Asti, M. Ghidini, R. Pellicelli, C. Pernechele, M. Solzi, F. Albertini, F. Casoli, S. Fabbrici, and L. Pareti, Phys. Rev. B 73, 094406 (2006) .

[15] S.-s. Yan, J. A. Barnard, F.-t Xu, J. L. Weston, and G. Zangari, Phys. Rev. B 64, 184403 (2001).

[16] G. Asti, M. Solzi, M. Ghidini, and F. M. Neri, Phys. Rev. B 69, 174401 (2004).

[17] G. Lavorato, E. Winkler, B. Rivas-Murias, and F. Rivadulla, Phys. Rev. B 94, 054405 (2016).

[18] N. L. Guo, G. P. Zhao, H. W. Zhang, X. L. Zhou, and Y. Deng, J. Magn. Magn. Mater. 323, 3049 (2011).

[19] R. S. Hassan, C. Mény, N. Viart, C. Ulhaq, G. Versini, J. L. Loison, and G. Pourroy, New J. Phys. 9, 364 (2007).

[20] N. Viart, R. S. Hassan, C. Mény, P. Panissod, C. Ulhaq-Bouillet, J. L. Loison, G. Versini, F. Huber, and G. Pourroy, Appl. Phys. Lett. 86, 192514 (2005).

[21] N. Viart, R. Sayed Hassan, C. Ulhaq-Bouillet, C. Mény, P. Panissod, J. L. Loison, G. Versini, F. Huber, G. Pourroy, J. Verbeeck, and G. Van Tendeloo, Acta Mater. 54, 191 (2006).

[22] I. S. Jurca, N. Viart, C. Mény, C. Ulhaq-Bouillet, P. Panissod, and G. Pourroy, Surf. Sci. 529, 215 (2003).

[23] M. Getzlaff, Fundamentals of Magnetism (Springer, New York, 2007).

[24] Q. Zhao, J. Chen, J. Wang, X. Zhang, G. Zhao, and Q. Ma, Sci. Rep. 7, 1 (2017).

[25] L. Aballe, M. Foerster, E. Pellegrin, J. Nicolas, and S. Ferrer, J. Synchrotron Radiat. 22, 745 (2015).

[26] A. Vansteenkiste, J. Leliaert, M. Dvornik, M. Helsen, F. GarciaSanchez, and B. Van Waeyenberge, AIP Adv. 4, 107133 (2014).

[27] R. Skomski and J. M. D. Coey, Phys. Rev. B 48, 15812 (1993).
[28] E. F. Kneller and R. Hawig, IEEE Trans. Magn. 27, 3588 (1991).

[29] M. Pousthomis, C. Garnero, C. G. Marcelot, T. Blon, S. Cayez, C. Cassignol, V. A. Du, M. Krispin, R. Arenal, K. Soulantica, G. Viau, and L. M. Lacroix, J. Magn. Magn. Mater. 424, 304 (2017).

[30] Y. Choi, J. S. Jiang, J. E. Pearson, and S. D. Bader, Appl. Phys. Lett. 91, 022502 (2007).

[31] Z. B. Li, B. G. Shen, and J. R. Sun, J. Appl. Phys. 113, 013902 (2013).

[32] D. S. Schmool, A. Apolinario, F. Casoli, and F. Albertini, IEEE Trans. Magn. 44, 3087 (2008).

[33] I. Panagiotopoulos, L. Withanawasam, and G. C. Hadjipanayis, J. Magn. Magn. Mater. 152, 353 (1996).

[34] P. Prieto, J. F. Marco, J. E. Prieto, S. Ruiz-gomez, L. Perez, R. P. Real, M. Vazquez, and J. de la Figuera, Appl. Surf. Sci. 436, 1067 (2018).

[35] K. V. O’Donovan, J. A. Borchers, S. Maat, M. J. Carey, and B. A. Gurney, J. Appl. Phys. 95, 7507 (2004).

[36] A. Hubert and R. Schäfer, Magnetic Domains (Springer-Verlag, Berlin, Heidelberg, 1998).

[37] See Supplemental Material at http://link.aps.org/ supplemental/10.1103/PhysRevB.98.214435, which includes Refs. [17,41,42].

[38] R. Pellicelli, M. Solzi, and C. Pernechele, J. Phys. D 47, 115002 (2014).

[39] S. A. Chambers, R. F. C. Farrow, S. Maat, M. F. Toney, L. Folks, J. G. Catalano, T. P. Trainor, and G. E. Brown, J. Magn. Magn. Mater. 246, 124 (2002).

[40] J.-B. Moussy, J. Phys. D 46, 143001 (2013).

[41] A. Jakobsson, E. Şasioğlu, P. Mavropoulos, M. Ležaić, B. Sanyal, G. Bihlmayer, and S. Blügel, Appl. Phys. Lett. 103, 102404 (2013).

[42] P. J. Jensen, H. Dreysse, and K. H. Bennemann, EPL 18, 463 (1992). 